\title{
Article \\ Influence of Metabolic Syndrome on Cancer Risk in HBV Carriers: A Nationwide Population Based Study Using the National Health Insurance Service Database
}

\author{
Jung Wan Choe ${ }^{1} \mathbb{D}$, Jong Jin Hyun ${ }^{1, *,+} \mathbb{D}^{\mathbb{C}}$, Bongseong Kim ${ }^{2}$ and Kyung-Do Han ${ }^{2, *,+}$ \\ 1 Department of Internal Medicine, Korea University Ansan Hospital, Ansan 15355, Korea; jwchoe@korea.ac.kr \\ 2 Department of Biostatistics, College of Medicine, The Catholic University of Korea, Seoul 06591, Korea; \\ qhdtjd12@gmail.com \\ * Correspondence: sean4h@korea.ac.kr (J.J.H.); hkd917@naver.com (K.-D.H.) \\ + These authors equally contributed as co-corresponder.
}

check for updates

Citation: Choe, J.W.; Hyun, J.J.; Kim, B.; Han, K.-D. Influence of Metabolic Syndrome on Cancer Risk in HBV Carriers: A Nationwide Population Based Study Using the National Health Insurance Service Database. J. Clin. Med. 2021, 10, 2401. https:// doi.org/10.3390/jcm10112401

Academic Editors: Hiroki Nishikawa and Emmanuel Andrès

Received: 26 April 2021

Accepted: 26 May 2021

Published: 29 May 2021

Publisher's Note: MDPI stays neutral with regard to jurisdictional claims in published maps and institutional affiliations.

Copyright: (c) 2021 by the authors. Licensee MDPI, Basel, Switzerland. This article is an open access article distributed under the terms and conditions of the Creative Commons Attribution (CC BY) license (https:/ / creativecommons.org/licenses/by/ $4.0 /)$.

\begin{abstract}
Purpose: Hepatitis B virus (HBV) infection and metabolic syndrome (MS) are known independent risk factors for hepatocellular carcinoma (HCC) and other extrahepatic organ malignancies. The purpose of this study was to investigate whether MS and HBV have synergistic effects on cancers and to examine whether increasing the number of MS components could lead to higher risk of cancer development. Materials and Methods: We evaluated data from 1,504,880 HBV-infected adults who underwent a regular HCC screening program provided by the Korean National Health Insurance Service between 2009 and 2016. Results: The prevalence of MS in Korean HBV patients was 38.7\% $(582,449 / 1,504,880)$. Among individuals with HBV infection, the presence of MS was associated with an increased risk for the majority of malignancies except for HCC ( $\mathrm{HR}=0.862, p$-value $<0.05)$. The presence of a higher number of MS components was associated with a significantly increased risk of developing cancers in most organs; only HCC was negatively associated with an increasing number of MS components $(p<0.01)$. Conclusions: Our data show that the presence of MS increases the risk for most malignancies, excluding HCC. Moreover, we found that as the number of MS components increased, the risk for most cancers also increased; this trend was reversed in HCC.
\end{abstract}

Keywords: metabolic syndrome; hepatitis B virus; neoplasms

\section{Introduction}

Hepatitis B virus (HBV) infection is known to play a role in chronic liver damage and the subsequent development of hepatocellular carcinoma (HCC) and cholangiocarcinoma [1,2]. While HBV is generally considered a hepatotropic virus, various studies have reported that HBV can exist in extrahepatic organs such as the pancreas, kidneys, skin, gastric mucosa, lymph nodes, spleen, bone marrow, colon, and testes [3]. Additionally, chronic HBV infection has been shown to increase the risk of extra-hepatic malignancies such as pancreatic cancer, gastric cancer, non-Hodgkin lymphoma and colon cancer [4].

Metabolic syndrome (MS), a condition that includes central obesity, hypertension, dyslipidemia, and diabetes, is a growing public health concern due to its high prevalence and poor long-term outcomes. While MS was initially only considered a risk factor for diabetes and cardiovascular disease, recent studies have suggested that it is also a risk factor for various types of cancer [5]. MS is known to be associated with a higher risk of liver, colorectal, and bladder cancers in men, and endometrial, pancreatic, colorectal, ovarian, and postmenopausal breast cancers in women. The pathophysiology that underlies the association of MS with cancer remains poorly understood to date. Moreover, whether MS as a whole carries a higher risk than each separate MS component is also unclear.

Despite the fact that there is a dearth of information about malignancies developing in extrahepatic sites as well as information describing which components of MS have 
synergistic effects on carcinogenesis, few studies have investigated the close association between the components of MS and HBV-related liver cirrhosis and HCC [6,7].

Therefore, the main objective of this study was to elucidate the synergistic association between MS and HBV in HCC and extrahepatic organ malignancies, and to investigate which MS components are associated with increased cancer risk.

\section{Materials}

\subsection{Study Population}

The National Health Insurance Service (NHIS) database, which is managed by the Korean government and covers approximately $97 \%$ of the Korean population, was used for this study [8]. Between 1 January 2009, and 31 December 2016, data on 2,567,812 HBVinfected adults over 40 years old were collected from the Korean National Liver Cancer Surveillance Program provided by NHIS. International Statistical Classification of Diseases and Related Health Problems, Tenth Revision (ICD-10) codes B16, B16.1, B16.2, B16.9, B17.0, B18.0 or B18.1 were used to select patients with HBV. Patients who received a diagnosis of HCC or other malignancies within 1 year of enrollment or had a history of malignancy at any time were excluded. Patients who failed to attend follow-up appointments and individuals who had missing data were also excluded. Finally, a total of 1,504,880 patients were included for this study (Figure 1). Presence of HCC was identified by ICD-10 code C22, and non-HCC cancers were also identified using ICD-10 codes as shown in Table S1.

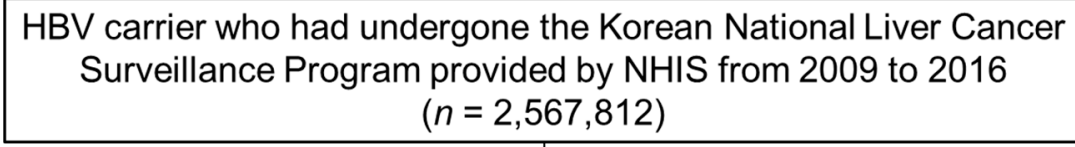

HBV patients included for analysis $(n=1,504,880)$

Figure 1. Flow chart of HBV-infected patients in the Korean NHIS database between 2009 and 2016. HBV, Hepatitis B virus; NHIS, National Health Insurance Service.

This study was approved by the Institutional Review Board of Korea University Ansan Hospital (No. 2019AS0012) and the research was conducted in accordance with the Helsinki declaration.

\subsection{Measurement of Variables}

During a Korean national health examination, waist circumference, body mass index, and systolic and diastolic pressure are typically measured. In addition, the levels of fasting plasma glucose, triglycerides (TG), total cholesterol, and high-density lipoprotein cholesterol (HDL-c) are also obtained. Lifestyle factors, such as smoking status, alcohol consumption, and physical activity, are also determined during the examination via a self-reported questionnaire. Smoking status is categorized as current, former, or never smokers. Alcohol intake is assessed by the frequency of ingestion and average amount of alcohol consumed each time. (non, mild (mean consumption $<30 \mathrm{~g} /$ day), or heavy (mean consumption $\geq 30 \mathrm{~g} /$ day)), Physical activity is determined by the number of times each week the subject exercises; exercising less than three times per week is defined as lacking physical activity. 


\subsection{Criteria of Metabolic Syndrome}

MS was defined as the presence of at least three of the following five criteria proposed by the American Heart Association and the National Heart, Lung and Blood Institute, together with the International Diabetes Federation, in 2009.

- Central obesity (waist circumference $>94 \mathrm{~cm}$ for males and $>80 \mathrm{~cm}$ for females), according to the Asian-Pacific criteria

- $\quad$ Raised TG $\geq 150 \mathrm{mg} / \mathrm{dL}(1.7 \mathrm{mmol} / \mathrm{L})$ or specific treatment for this lipid abnormality

- Reduced HDL-c < $40 \mathrm{mg} / \mathrm{dL}(1.03 \mathrm{mmol} / \mathrm{L})$ in males and $<50 \mathrm{mg} / \mathrm{dL}(1.29 \mathrm{mmol} / \mathrm{L})$ in females or specific treatment for this lipid abnormality

- $\quad$ Raised blood pressure, systolic $\geq 130$ or diastolic $\geq 85 \mathrm{~mm} \mathrm{Hg}$, or treatment of previously diagnosed hypertension

- Raised fasting plasma glucose $\geq 100 \mathrm{mg} / \mathrm{dL}$ (5.6 mmol/L), or previously diagnosed type 2 diabetes.

\subsection{Statistical Analysis}

SAS System version 9.4 for Windows was used for all statistical analyses. All categorical variables are expressed as percentages and all continuous variables are expressed as mean \pm standard deviation. Multivariate Cox regression analysis was performed to examine the hazard ratio (HR) and confidence interval for the relationship between MS status and malignant disease. The multivariable analyses were adjusted for factors including age, sex, body mass index, smoking status, alcohol consumption and physical activity, $p$ values $<0.05$ were considered statistically significant.

\section{Results}

The prevalence of MS in Korean HBV patients was 38.7\% (582,449/1,504,880).Table 1 shows baseline characteristics of the study population. The median age of the overall study population was 53 years. Patients with MS tended to be older and obese, consumed significantly more alcohol, and had a higher proportion of MS components. Among individuals infected with $\mathrm{HBV}$, the presence of MS was associated with an increased risk of developing malignancies other than HCC (Figure 2, Table 2). As for HCC, the relative risk of incidence rate was 0.862 , which was remarkably lower in HBV carriers with MS than those without (3.57 vs. $4.02, p<0.0001)$. The cumulative incidence of overall malignancy was significantly higher in HBV carriers with MS than in those without MS (14.21 vs. 12.20, $p<0.001$ ), however, this trend was reversed for HCC (Figure 3).

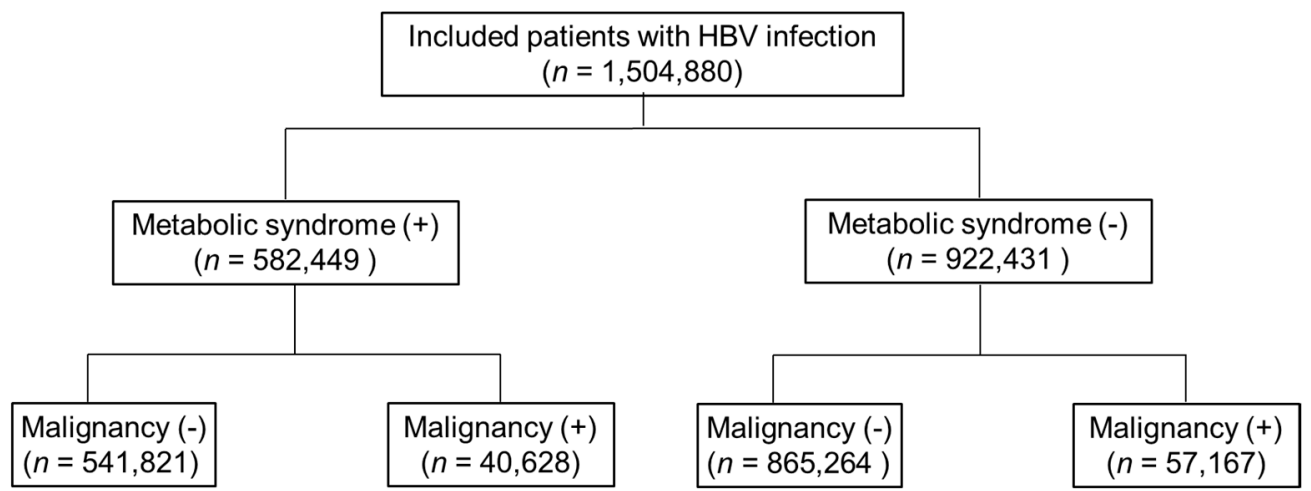

Figure 2. Diagram for incidences of metabolic syndrome and malignancy in HBV-infected patients. HBV, Hepatitis B virus. 
Table 1. Characteristics of the study population.

\begin{tabular}{|c|c|c|c|}
\hline Characteristics & Overall $(n=1,504,880)$ & $\begin{array}{l}\text { Metabolic Syndrome }(+) \\
\quad(n=582,449)\end{array}$ & $\begin{array}{c}\text { Metabolic Syndrome }(-) \\
\quad(n=922,431)\end{array}$ \\
\hline $\begin{array}{l}\text { Age, mean, years } \\
\text { Sex }\end{array}$ & $53.5 \pm 9.77$ & $56.4 \pm 9.88$ & $51.55 \pm 9.52$ \\
\hline $\begin{array}{l}\text { Men } \\
\text { Smoke }\end{array}$ & $846,687(56.3)$ & $496,146(53.79)$ & $350,541(60.18)$ \\
\hline $\begin{array}{l}\text { Non/Ex/Current } \\
\text { Alcohol }\end{array}$ & $57.7 \% / 18.9 \% / 23.4 \%$ & $54.2 \% / 21.3 \% / 24.5 \%$ & $59.8 \% / 17.5 \% / 22.7 \%$ \\
\hline Non/Mild/Heavy & $55.9 \% / 33.9 \% / 10.2 \%$ & $54.7 \% / 32.6 \% / 12.7 \%$ & $56.6 \% / 34.8 \% / 8.6 \%$ \\
\hline Physical activity & $320,665(21.3)$ & $122,217(21.0)$ & $198,448(21.5)$ \\
\hline $\begin{array}{c}\text { BMI } \\
\text { Metabolic syndrome factors }\end{array}$ & $24.2 \pm 3.01$ & $25.8 \pm 3.28$ & $23.32 \pm 2.83$ \\
\hline $\begin{array}{l}\text { Waist circumference }>94 \mathrm{~cm} \text { for males } \\
\text { and }>80 \mathrm{~cm} \text { for females }\end{array}$ & $405,984(27.0)$ & $176,482(30.3)$ & $229,502(24.9)$ \\
\hline $\begin{array}{c}\text { Systolic } \geq 130 \text { or diastolic } \geq 85 \mathrm{~mm} \mathrm{Hg} \\
\text { or diagnosed HTN }\end{array}$ & $595,191(39.6)$ & $388,646(66.7)$ & $206,545(22.4)$ \\
\hline $\begin{array}{c}\text { Fasting plasma glucose } \geq 100 \mathrm{mg} / \mathrm{dL} \\
\text { or type } 2 \mathrm{DM}\end{array}$ & 284,403 (18.9) & $187,548(32.2)$ & $96,855(10.5)$ \\
\hline $\begin{array}{l}\text { HDL-c }<40 \mathrm{mg} / \mathrm{dL} \text { in males and } \\
\quad<50 \mathrm{mg} / \mathrm{dL} \text { in females }\end{array}$ & $604,455(40.2)$ & $327,155(56.2)$ & $277,300(30.1)$ \\
\hline $\mathrm{TG} \geq 150 \mathrm{mg} / \mathrm{dL}$ & $645,159(42.9)$ & $315,442(54.2)$ & 329,717 (35.7) \\
\hline
\end{tabular}

$n$ (\%), BMI; body mass index, HTN; hypertension, TG, triglycerides; HDL-c, high-density lipoprotein cholesterol.

Table 2. Relationship between malignant neoplasms and the presence of metabolic syndrome components in HBVinfected patients.

\begin{tabular}{|c|c|c|c|c|c|c|}
\hline \multirow[t]{2}{*}{ Cancer Site } & \multicolumn{2}{|c|}{$\begin{array}{c}\text { MS(+) } \\
(n=582,449)\end{array}$} & \multicolumn{2}{|c|}{$\begin{array}{c}\text { MS }(-) \\
(n=922,431)\end{array}$} & \multirow[b]{2}{*}{ Adjusted HR } & \multirow[b]{2}{*}{$p$-Value } \\
\hline & Case, No. & $\begin{array}{c}\text { Incidence Rate } \\
\text { No./1000 PYs }\end{array}$ & Case, No. & $\begin{array}{c}\text { Incidence Rate } \\
\text { No./1000 PYs }\end{array}$ & & \\
\hline Overall cancer & 40,628 & 14.21 & 57,167 & 12.20 & $1.17(1.15-1.18)$ & $<0.0001$ \\
\hline HCC & 10,156 & 3.57 & 19,256 & 4.02 & $0.86(0.84-0.88)$ & $<0.0001$ \\
\hline Biliary & 1312 & 0.44 & 1489 & 0.31 & $1.45(1.35-1.56)$ & $<0.0001$ \\
\hline Stomach & 5022 & 1.71 & 5638 & 1.17 & $1.46(1.41-1.52)$ & $<0.0001$ \\
\hline Colorectum & 4260 & 1.45 & 4473 & 0.93 & $1.56(1.50-1.63)$ & $<0.0001$ \\
\hline Esophagus & 634 & 0.21 & 783 & 0.16 & $1.33(1.20-1.48)$ & $<0.0001$ \\
\hline Pancreas & 1468 & 0.50 & 1408 & 0.29 & $1.72(1.60-1.85)$ & $<0.0001$ \\
\hline lung & 4260 & 1.44 & 4937 & 1.02 & $1.42(1.36-1.48)$ & $<0.0001$ \\
\hline Renal & 1072 & 0.36 & 943 & 0.20 & $1.87(1.71-2.04)$ & $<0.0001$ \\
\hline thyroid & 2985 & 1.01 & 4744 & 0.98 & $1.03(0.98-1.08)$ & 0.29 \\
\hline Laryngeal & 333 & 0.11 & 369 & 0.08 & $1.48(1.28-1.71)$ & $<0.0001$ \\
\hline Oral & 614 & 0.21 & 845 & 0.17 & 1.19 (1.07-1.32) & 0.001 \\
\hline Lymphoma & 826 & 0.28 & 1158 & 0.24 & 1.17 (1.07-1.28) & 0.006 \\
\hline Leukemia & 443 & 0.15 & 530 & 0.11 & $1.38(1.21-1.56)$ & $<0.0001$ \\
\hline Skin & 140 & 0.05 & 168 & 0.03 & $1.38(1.10-1.72)$ & 0.005 \\
\hline $\begin{array}{c}\text { Nerves } \\
\text { Male }\end{array}$ & 432 & 0.15 & 559 & 0.12 & $1.27(1.12-1.44)$ & 0.0002 \\
\hline Prostate & 2785 & 1.58 & 2911 & 1.12 & $1.43(1.36-1.50)$ & $<0.0001$ \\
\hline $\begin{array}{c}\text { Testicular } \\
\text { Female }\end{array}$ & 30 & 0.02 & 33 & 0.01 & $1.34(0.82-2.20)$ & 0.24 \\
\hline Breast & 1717 & 1.45 & 2215 & 1.43 & 1.01 (0.95-1.08) & 0.67 \\
\hline Corpus & 286 & 0.24 & 489 & 0.22 & $1.10(0.95-1.28)$ & 0.19 \\
\hline Ovarian & 254 & 0.21 & 458 & 0.21 & $1.04(0.89-1.22)$ & 0.59 \\
\hline Cervical & 257 & 0.22 & 456 & 0.20 & $1.06(0.91-1.23)$ & 0.47 \\
\hline
\end{tabular}



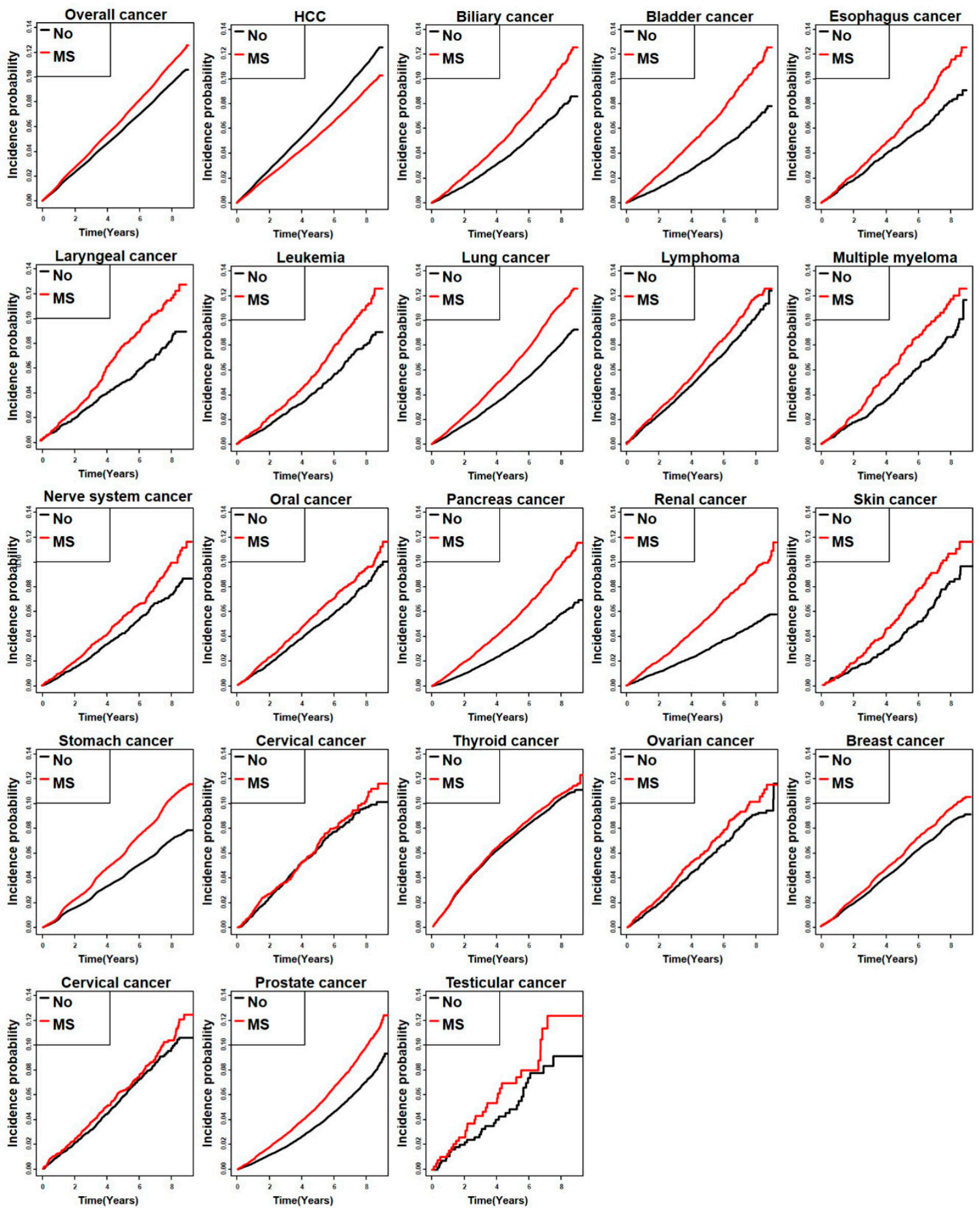

Figure 3. Cumulative incidence of cancer according to metabolic syndrome status in HBV-infected patients. MS, Metabolic syndrome; HCC, hepatocellular carcinoma.

Table 3 shows the relative malignant neoplasm risk associated with five metabolic factors. Among the components of the MS, high serum TG and low HDL-c levels were negative contributing factors for HCC. On the other hand, the two aforementioned components were independent risk factors for malignancy in other organs. Additionally, increased waist circumference, high blood pressure, and high blood glucose level in HBV patients were positive contributing factors for all types of cancers including HCC (Table 3). 
Table 3. Effect of each metabolic syndrome component on malignant neoplasm risk.

\begin{tabular}{|c|c|c|c|c|c|c|}
\hline Risk Factors & Overall Cancer & Liver & Biliary & Bladder & Colorectal & Esophagus \\
\hline $\begin{array}{c}\text { Serum high TG } \geq \\
150 \text { vs. }<150\end{array}$ & $1.13(0.99-1.28)$ & $0.46(0.45-0.47)$ & $1.16(1.07-1.25)$ & $1.46(1.34-1.59)$ & $1.29(1.24-1.35)$ & $1.21(1.09-1.34)$ \\
\hline $\begin{array}{c}\text { Serum low HDL } \\
\text { cholesterol }<40 \\
\text { vs. } \geq 40\end{array}$ & $1.01(1.00-1.02)$ & $0.68(0.66-0.70)$ & $1.20(1.11-1.29)$ & $1.16(1.07-1.26)$ & $1.16(1.11-1.21)$ & $0.88(0.80-1.01)$ \\
\hline $\begin{array}{c}\text { Serum abnormal } \\
\text { glucose } \geq 100 \\
\text { vs. }<100\end{array}$ & $1.31(1.30-1.33)$ & $1.53(1.49-1.56)$ & $1.52(1.41-1.63)$ & $1.72(1.58-1.87)$ & $1.53(1.47-1.60)$ & $1.80(1.62-2.00)$ \\
\hline $\begin{array}{l}\text { Central obesity } \\
\text { Yes vs. No }\end{array}$ & $1.20(1.18-1.21)$ & $1.20(1.18-1.23)$ & $1.30(1.20-1.41)$ & $1.41(1.29-1.54)$ & $1.36(1.31-1.43)$ & $0.99(0.88-1.11)$ \\
\hline \multirow{2}{*}{$\begin{array}{c}\text { History of } \\
\text { hypertension Yes } \\
\text { vs. No }\end{array}$} & $1.42(1.41-1.44)$ & $1.44(1.40-1.47)$ & $1.86(1.72-2.01)$ & $2.06(1.88-2.27)$ & $1.76(1.68-1.84)$ & $1.98(1.76-2.21)$ \\
\hline & Laryngeal & Leukemia & Lung & Lymphoma & Multiplemyeloma & Nerves \\
\hline $\begin{array}{l}\text { Serum high TG } \geq \\
150 \text { vs. }<150\end{array}$ & $1.44(1.24-1.67)$ & $1.12(0.99-1.27)$ & $1.25(1.20-1.31)$ & $0.95(0.87-1.04)$ & $1.03(0.88-1.21)$ & $1.14(1.01-1.29)$ \\
\hline $\begin{array}{l}\text { Serum low HDL } \\
\text { cholesterol }<40 \\
\text { vs. } \geq 40\end{array}$ & $0.91(0.78-1.06)$ & $1.28(1.13-1.45)$ & $1.11(1.07-1.16)$ & $1.12(1.02-1.22)$ & $1.37(1.17-1.60)$ & $1.13(0.99-1.28)$ \\
\hline $\begin{array}{l}\text { Serum abnormal } \\
\text { glucose } \geq 100 \text { vs. } \\
\quad<100\end{array}$ & $1.86(1.60-2.16)$ & $1.19(1.05-1.34)$ & $1.48(1.42-1.54)$ & $1.21(1.11-1.32)$ & $1.19(1.01-1.39)$ & $1.13(0.99-1.28)$ \\
\hline $\begin{array}{l}\text { Central obesity } \\
\text { Yes vs. No }\end{array}$ & $0.98(0.83-1.15)$ & $1.21(1.06-1.39)$ & $1.10(1.05-1.15)$ & $1.23(1.12-1.35)$ & $1.44(1.22-1.69)$ & $1.22(1.06-1.39)$ \\
\hline \multirow[t]{2}{*}{$\begin{array}{c}\text { History of } \\
\text { hypertension Yes } \\
\text { vs. No }\end{array}$} & $1.93(1.64-2.27)$ & $1.31(1.15-1.49)$ & $1.75(1.67-1.83)$ & $1.28(1.17-1.40)$ & $1.68(1.42-1.98)$ & $1.44(1.27-1.64)$ \\
\hline & Oral & Pancreas & Renal & Skin & Stomach & Thyroid \\
\hline $\begin{array}{c}\text { Serum high } \mathrm{TG} \geq \\
150 \text { vs. }<150\end{array}$ & $1.12(1.01-1.25)$ & $1.29(1.19-1.38)$ & $1.44(1.32-1.57)$ & $1.16(0.93-1.46)$ & $1.19(1.15-1.24)$ & $1.03(0.98-1.08)$ \\
\hline $\begin{array}{l}\text { Serum low HDL } \\
\text { cholesterol }<40 \\
\text { vs. } \geq 40\end{array}$ & $0.96(0.86-1.06)$ & $1.33(1.23-1.43)$ & $1.31(1.20-1.43)$ & $1.18(0.94-1.48)$ & $1.05(1.01-1.09)$ & $1.17(1.12-1.23)$ \\
\hline $\begin{array}{l}\text { Serum abnormal } \\
\text { glucose } \geq 100 \text { vs. } \\
\quad<100\end{array}$ & $1.34(1.21-1.48)$ & $1.81(1.68-1.95)$ & $1.46(1.34-1.59)$ & $1.14(0.91-1.42)$ & $1.53(1.47-1.59)$ & $1.00(0.96-1.05)$ \\
\hline $\begin{array}{c}\text { Central obesity } \\
\text { Yes vs. No }\end{array}$ & $0.97(0.86-1.09)$ & $1.33(1.23-1.44)$ & $1.57(1.44-1.72)$ & $1.33(1.05-1.69)$ & $1.28(1.23-1.34)$ & $1.08(1.03-1.14)$ \\
\hline \multirow[t]{2}{*}{$\begin{array}{c}\text { History of } \\
\text { hypertension Yes } \\
\text { vs. No }\end{array}$} & $1.51(1.36-1.68)$ & $1.83(1.69-1.98)$ & $2.24(2.03-2.47)$ & $1.66(1.31-2.11)$ & $1.62(1.56-1.69)$ & $0.92(0.88-0.96)$ \\
\hline & Cervical & Ovarian & Corpus & Breast & Prostate & Testicular \\
\hline $\begin{array}{c}\text { Serum high } T G \geq \\
150 \text { vs. }<150\end{array}$ & $0.88(0.75-1.02)$ & $1.04(0.90-1.21)$ & $0.95(0.82-1.10)$ & $1.04(0.98-1.11)$ & $1.11(1.05-1.17)$ & $1.36(0.83-2.24)$ \\
\hline $\begin{array}{l}\text { Serum low HDL } \\
\text { cholesterol }<40 \\
\text { vs. } \geq 40\end{array}$ & $0.92(0.80-1.07)$ & $0.96(0.83-1.12)$ & $1.01(0.88-1.16)$ & $1.03(0.98-1.09)$ & $1.35(1.28-1.43)$ & $0.99(0.59-1.67)$ \\
\hline $\begin{array}{l}\text { Serum abnormal } \\
\text { glucose } \geq 100 \mathrm{vs} \\
\quad<100\end{array}$ & $1.17(1.01-1.37)$ & $1.02(0.88-1.20)$ & $1.02(0.88-1.18)$ & $0.96(0.91-1.02)$ & $1.15(1.09-1.21)$ & $1.17(0.71-1.91)$ \\
\hline $\begin{array}{l}\text { Central obesity } \\
\text { Yes vs. No }\end{array}$ & $1.31(1.11-1.54)$ & $1.20(1.02-1.42)$ & $1.43(1.23-1.67)$ & $1.00(0.94-1.07)$ & $1.26(1.19-1.33)$ & $1.82(1.11-3.00)$ \\
\hline $\begin{array}{c}\text { History of } \\
\text { hypertension Yes } \\
\text { vs. No }\end{array}$ & $1.12(0.96-1.29)$ & $0.99(0.85-1.15)$ & $1.06(0.92-1.22)$ & $1.06(1.00-1.13)$ & $1.90(1.80-2.02)$ & $1.19(0.72-1.99)$ \\
\hline
\end{tabular}

TG, triglycerides; HDL, high-density lipoprotein.

The relationship between malignant neoplasms and the number of individual MS components is shown in Table 4. The presence of a higher number of MS components was associated with a significantly increased risk of developing cancers in most organs; only HCC was negatively associated with an increased number of MS components. 
Table 4. Relationship between malignant neoplasms and the number of individual metabolic syndrome components.

\begin{tabular}{|c|c|c|c|c|c|c|}
\hline No. of MS Component & No. of Patients & Event & Duration & Rate & HR (95\% CI) & $p$-Value for Trend \\
\hline \multicolumn{7}{|l|}{ Overall cancers } \\
\hline 0 & 259,650 & 12,979 & $1,319,346.67$ & 9.84 & 1(Ref.) & $<0.0001$ \\
\hline 1 & 334,595 & 21,185 & $1,711,059.87$ & 12.38 & $1.26(1.23-1.29)$ & \\
\hline 2 & 328,186 & 23,003 & $1,656,449.03$ & 13.89 & $1.41(1.38-1.44)$ & \\
\hline 3 & 285,443 & 19,953 & $1,420,455.64$ & 14.05 & $1.43(1.39-1.46)$ & \\
\hline 4 & 207,646 & 14,238 & $1,012,356.88$ & 14.06 & 1.43 (1.40-1.47) & \\
\hline 5 & 89,360 & 6437 & $426,614.01$ & 15.09 & $1.54(1.50-1.59)$ & \\
\hline Liver & & & & & & $<0.0001$ \\
\hline 0 & 259,650 & 4018 & $1,345,045.29$ & 2.99 & 1(Ref.) & \\
\hline 1 & 334,595 & 7392 & $2,345,505.60$ & 3.15 & $1.06(1.02-1.10)$ & \\
\hline 2 & 328,186 & 7846 & $2,680,088.93$ & 2.93 & $1.00(0.94-1.02)$ & \\
\hline 3 & 285,443 & 5581 & $2,521,278.33$ & 2.21 & $0.74(0.71-0.77)$ & \\
\hline 4 & 207,646 & 3175 & $1,960,972.12$ & 1.62 & $0.54(0.52-0.57)$ & \\
\hline 5 & 89,360 & 1400 & $920,740.46$ & 1.52 & $0.51(0.48-0.54)$ & \\
\hline Biliary & & & & & & $<0.0001$ \\
\hline 0 & 259,650 & 250 & $1,354,435.46$ & 0.18 & 1(Ref.) & \\
\hline 1 & 334,595 & 545 & $1,765,727.59$ & 0.31 & $1.67(1.44-1.94)$ & \\
\hline 2 & 328,186 & 694 & $1,713,856.28$ & 0.40 & $2.19(1.90-2.53)$ & \\
\hline 3 & 285,443 & 606 & $1,469,120.76$ & 0.41 & $2.24(1.94-2.60)$ & \\
\hline 4 & 207,646 & 475 & $1,046,063.01$ & 0.45 & $2.48(2.13-2.89)$ & \\
\hline 5 & 89,360 & 231 & $441,763.87$ & 0.52 & $2.87(2.40-3.43)$ & \\
\hline Bladder & & & & & & $<0.0001$ \\
\hline 0 & 259,650 & 172 & $1,354,382.24$ & 0.13 & 1(Ref.) & \\
\hline 1 & 334,595 & 386 & $1,765,390$ & 0.22 & $1.72(1.44-2.06)$ & \\
\hline 2 & 328,186 & 523 & $1,713,593.9$ & 0.31 & $2.40(2.02-2.85)$ & \\
\hline 3 & 285,443 & 494 & $1,468,657.14$ & 0.34 & $2.66(2.23-3.16)$ & \\
\hline 4 & 207,646 & 425 & $1,045,757.91$ & 0.41 & $3.22(2.70-3.85)$ & \\
\hline 5 & 89,360 & 199 & $441,566.99$ & 0.45 & $3.59(2.93-4.40)$ & \\
\hline Esophagus & & & & & & $<0.0001$ \\
\hline 0 & 259,650 & 135 & $1,354,496.91$ & 0.10 & 1(Ref.) & \\
\hline 1 & 334,595 & 197 & $1,765,958.15$ & 0.11 & $1.11(0.91-1.37)$ & \\
\hline 2 & 328,186 & 220 & $1,714,148.16$ & 0.13 & $1.29(1.05-1.57)$ & \\
\hline 3 & 285,443 & 194 & $1,469,416.35$ & 0.13 & $1.32(1.07-1.63)$ & \\
\hline 4 & 207,646 & 193 & $1,046,368.28$ & 0.15 & 1.50 (1.19-1.85) & \\
\hline 5 & 89,360 & 66 & $441,912.08$ & 0.15 & $1.50(1.11-2.01)$ & \\
\hline Laryngeal & & & & & & $<0.0001$ \\
\hline 0 & 259,650 & 52 & $1,354,694.77$ & 0.04 & 1(Ref.) & \\
\hline 1 & 334,595 & 140 & $1,766,135.1$ & 0.08 & $2.06(1.50-2.84)$ & \\
\hline 2 & 328,186 & 177 & 1714418.44 & 0.10 & $2.69(1.97-3.66)$ & \\
\hline 3 & 285,443 & 162 & $1,469,549.65$ & 0.11 & $2.79(1.88-4.13)$ & \\
\hline 4 & 207,646 & 112 & $1,046,543.83$ & 0.11 & $2.80(2.01-3.89)$ & \\
\hline 5 & 89,360 & 53 & $441,937.8$ & 0.12 & $3.09(2.27-4.21)$ & \\
\hline Leukemia & & & & & & $<0.0001$ \\
\hline 0 & 259,650 & 129 & $1,354,546.93$ & 0.1 & 1(Ref.) & \\
\hline 1 & 334,595 & 189 & $1,766,169.07$ & 0.11 & $1.12(0.90-1.40)$ & \\
\hline 2 & 328,186 & 212 & $1,714,518.59$ & 0.12 & $1.30(1.04-1.62)$ & \\
\hline 3 & 285,443 & 221 & $1,469,627.1$ & 0.15 & 1.59 (1.28-1.97) & \\
\hline 4 & 207,646 & 166 & $1,046,523.58$ & 0.16 & $1.68(1.34-2.12)$ & \\
\hline 5 & 89,360 & 75 & $441,976.02$ & 0.17 & $1.80(1.50-2.13)$ & \\
\hline
\end{tabular}


Table 4. Cont.

\begin{tabular}{|c|c|c|c|c|c|c|}
\hline No. of MS Component & No. of Patients & Event & Duration & Rate & HR (95\% CI) & $p$-Value for Trend \\
\hline Lung & & & & & & $<0.0001$ \\
\hline 0 & 259,650 & 963 & $1,353,109.74$ & 0.71 & 1(Ref.) & \\
\hline 1 & 334,595 & 1816 & $1,763,511.24$ & 1.03 & $1.44(1.34-1.56)$ & \\
\hline 2 & 328,186 & 2158 & $1,711,355.12$ & 1.26 & 1.77 (1.64-1.91) & \\
\hline 3 & 285,443 & 2007 & $1,466,855.44$ & 1.37 & $1.93(1.79-2.08)$ & \\
\hline 4 & 207,646 & 1562 & $1,044,462.24$ & 1.50 & $2.12(1.96-2.30)$ & \\
\hline 5 & 89,360 & 691 & $441,013.88$ & 1.57 & $2.23(2.02-2.46)$ & \\
\hline Lymphoma & & & & & & $<0.0001$ \\
\hline 0 & 259,650 & 268 & $1,354,169.19$ & 0.20 & 1(Ref.) & \\
\hline 1 & 334,595 & 413 & $1,765,390.75$ & 0.23 & $1.18(1.01-1.38)$ & \\
\hline 2 & 328,186 & 477 & $1,713,784.81$ & 0.28 & $1.38(1.17-1.63)$ & \\
\hline 3 & 285,443 & 411 & $1,469,064.72$ & 0.28 & $1.41(1.21-1.63)$ & \\
\hline 4 & 207,646 & 301 & $1,046,276.88$ & 0.29 & $1.42(1.21-1.65)$ & \\
\hline 5 & 89,360 & 131 & $441,755.53$ & 0.29 & $1.51(1.22-1.86)$ & \\
\hline Multiple myeloma & & & & & & $<0.0001$ \\
\hline 0 & 259,650 & 73 & $1,354,665.95$ & 0.05 & 1(Ref.) & \\
\hline 1 & 334,595 & 110 & $1,766,257.7$ & 0.06 & $1.15(0.86-1.55)$ & \\
\hline 2 & 328,186 & 155 & $1,714,582.95$ & 0.09 & $1.68(1.27-2.21)$ & \\
\hline 3 & 285,443 & 140 & $1,469,721.72$ & 0.10 & $1.77(1.33-2.35)$ & \\
\hline 4 & 207,646 & 105 & $1,046,641.76$ & 0.10 & $1.86(1.42-2.52)$ & \\
\hline 5 & 89,360 & 55 & $441,975.23$ & 0.12 & $2.33(1.64-3.31)$ & \\
\hline Nerves & & & & & & $<0.0001$ \\
\hline 0 & 259,650 & 114 & $1,354,590.62$ & 0.08 & 1(Ref.) & \\
\hline 1 & 334,595 & 214 & $1,766,091.27$ & 0.12 & $1.44(1.15-1.80)$ & \\
\hline 2 & 328,186 & 231 & $1,714,539.85$ & 0.13 & $1.60(1.28-2.00)$ & \\
\hline 3 & 285,443 & 168 & $1,469,699.8$ & 0.14 & $1.68(1.24-2.29)$ & \\
\hline 4 & 207,646 & 151 & $1,046,570.14$ & 0.14 & $1.73(1.35-2.20)$ & \\
\hline 5 & 89,360 & 66 & $441,961.96$ & 0.15 & $1.78(1.42-2.23)$ & \\
\hline Oral & & & & & & $<0.0001$ \\
\hline 0 & 259,650 & 157 & $1,354,488.07$ & 0.12 & 1(Ref.) & \\
\hline 1 & 334,595 & 320 & $1,765,725.54$ & 0.18 & $1.57(1.20-2.10)$ & \\
\hline 2 & 328,186 & 318 & $1,714,009.71$ & 0.19 & $1.60(1.30-1.90)$ & \\
\hline 3 & 285,443 & 295 & $1,469,253.33$ & 0.20 & $1.74(1.41-2.14)$ & \\
\hline 4 & 207,646 & 220 & $1,046,308.21$ & 0.21 & $1.81(1.50-2.19)$ & \\
\hline 5 & 89,360 & 97 & $441,892.7$ & 0.22 & $1.90(1.57-2.30)$ & \\
\hline Pancreatic & & & & & & $<0.0001$ \\
\hline 0 & 259,650 & 240 & $1,354,462.12$ & 0.18 & 1(Ref.) & \\
\hline 1 & 334,595 & 521 & $1,765,820.13$ & 0.30 & $1.66(1.43-1.94)$ & \\
\hline 2 & 328,186 & 647 & $1,714,141.81$ & 0.38 & $2.13(1.84-2.47)$ & \\
\hline 3 & 285,443 & 668 & $1,469,134.67$ & 0.45 & $2.58(2.22-2.98)$ & \\
\hline 4 & 207,646 & 553 & $1,046,195.85$ & 0.53 & $3.01(2.59-3.50)$ & \\
\hline 5 & 89,360 & 247 & $441,812.98$ & 0.56 & $3.19(2.67-3.82)$ & \\
\hline Renal & & & & & & $<0.0001$ \\
\hline 0 & 259,650 & 185 & $1,354,295.16$ & 0.14 & 1(Ref.) & \\
\hline 1 & 334,595 & 345 & $1,765,540.94$ & 0.20 & $1.43(1.20-1.71)$ & \\
\hline 2 & 328,186 & 413 & $1,713,765.23$ & 0.24 & $1.76(1.48-2.10)$ & \\
\hline 3 & 285,443 & 460 & $1,468,691.95$ & 0.31 & $2.30(1.94-2.72)$ & \\
\hline 4 & 207,646 & 412 & $1,045,740.36$ & 0.39 & $2.90(2.44-3.44)$ & \\
\hline 5 & 89,360 & 200 & $441,542.37$ & 0.45 & $3.34(2.73-4.08)$ & \\
\hline
\end{tabular}


Table 4. Cont.

\begin{tabular}{|c|c|c|c|c|c|c|}
\hline No. of MS Component & No. of Patients & Event & Duration & Rate & HR (95\% CI) & $p$-Value for Trend \\
\hline Skin & & & & & & 0.0056 \\
\hline 0 & 259,650 & 37 & $1,354,723.35$ & 0.03 & 1(Ref.) & \\
\hline 1 & 334,595 & 54 & $1,766,370.81$ & 0.03 & $1.12(0.73-1.70)$ & \\
\hline 2 & 328,186 & 70 & $1,714,708.36$ & 0.04 & $1.52(0.98-2.36)$ & \\
\hline 3 & 285,443 & 66 & $1,469,802.02$ & 0.04 & $1.65(1.11-2.43)$ & \\
\hline 4 & 207,646 & 52 & $1,046,697.71$ & 0.05 & $1.83(1.23-2.72)$ & \\
\hline 5 & 89,360 & 24 & $442,015.79$ & 0.05 & $2.02(1.21-3.38)$ & \\
\hline Stomach & & & & & & $<0.0001$ \\
\hline 0 & 259,650 & 1176 & $1,351,380.7$ & 0.87 & 1(Ref.) & \\
\hline 1 & 334,595 & 2056 & $1,760,563.77$ & 1.17 & $1.34(1.25-1.44)$ & \\
\hline 2 & 328,186 & 2406 & $1,707,972.12$ & 1.41 & $1.62(1.51-1.74)$ & \\
\hline 3 & 285,443 & 2411 & $1,463,287.18$ & 1.65 & $1.90(1.77-2.04)$ & \\
\hline 4 & 207,646 & 1815 & $1,041,833.59$ & 1.74 & $2.01(1.87-2.16)$ & \\
\hline 5 & 89,360 & 796 & $439,998.98$ & 1.81 & $2.09(1.91-2.29)$ & \\
\hline Thyroid & & & & & & 0.0001 \\
\hline 0 & 259,650 & 1566 & $1,348,965.02$ & 1.16 & 1(Ref.) & \\
\hline 1 & 334,595 & 1914 & $1,420,102.54$ & 1.35 & $1.16(1.09-1.24)$ & \\
\hline 2 & 328,186 & 1760 & $1,207,075.11$ & 1.46 & $1.26(1.17-1.35)$ & \\
\hline 3 & 285,443 & 1503 & $980,091.68$ & 1.53 & $1.32(1.23-1.42)$ & \\
\hline 4 & 207,646 & 1025 & $668,392.53$ & 1.53 & $1.32(1.22-1.44)$ & \\
\hline 5 & 89,360 & 457 & $240,327.73$ & 1.90 & $1.41(1.27-1.57)$ & \\
\hline Cervical & & & & & & 0.26 \\
\hline 0 & 147,274 & 152 & $758,779.63$ & 0.20 & 1(Ref.) & \\
\hline 1 & 149,487 & 171 & $787,486.11$ & 0.22 & $1.09(0.87-1.35)$ & \\
\hline 2 & 129,524 & 127 & $678,426.82$ & 0.19 & $0.93(0.73-1.19)$ & \\
\hline 3 & 113,076 & 114 & $584,045.39$ & 0.20 & $0.98(0.78-1.24)$ & \\
\hline 4 & 82,287 & 102 & $418,459.67$ & 0.24 & $1.22(0.95-1.57)$ & \\
\hline 5 & 36,545 & 46 & $182,169.80$ & 0.25 & $1.26(0.91-1.75)$ & \\
\hline Ovarian & & & & & & 0.57 \\
\hline 0 & 147,274 & 163 & $758,835.14$ & 0.21 & 1(Ref.) & \\
\hline 1 & 149,487 & 157 & $787,573.26$ & 0.20 & $0.93(0.75-1.16)$ & \\
\hline 2 & 129,524 & 138 & $678,527.99$ & 0.20 & $0.95(0.76-1.19)$ & \\
\hline 3 & 113,076 & 118 & $584,111.02$ & 0.20 & $0.94(0.74-1.19)$ & \\
\hline 4 & 82,287 & 87 & $418,548.71$ & 0.21 & $0.97(0.75-1.26)$ & \\
\hline 5 & 36,545 & 49 & $182,202.54$ & 0.27 & $1.26(0.91-1.73)$ & \\
\hline Corpus & & & & & & 0.45 \\
\hline 0 & 147,274 & 168 & $758,785.66$ & 0.22 & 1(Ref.) & \\
\hline 1 & 149,487 & 174 & $787,526.35$ & 0.22 & $1.00(0.81-1.23)$ & \\
\hline 2 & 129,524 & 147 & $678,420.86$ & 0.22 & $1.00(0.78-1.22)$ & \\
\hline 3 & 113,076 & 133 & $584,041.72$ & 0.23 & $1.03(0.82-1.29)$ & \\
\hline 4 & 82,287 & 99 & $418,475.40$ & 0.24 & $1.07(0.84-1.37)$ & \\
\hline 5 & 36,545 & 54 & $182,165.06$ & 0.30 & 1.35 (0.99-1.83) & \\
\hline Breast & & & & & & $<0.0001$ \\
\hline 0 & 147,274 & 1308 & $755,595.15$ & 1.73 & 1(Ref.) & \\
\hline 1 & 149,487 & 1244 & $724,519.51$ & 1.72 & $0.99(0.92-1.07)$ & \\
\hline 2 & 129,524 & 1030 & $57,438.09$ & 1.79 & $1.04(0.95-1.13)$ & \\
\hline 3 & 113,076 & 834 & $460,595.34$ & 1.81 & $1.05(0.96-1.15)$ & \\
\hline 4 & 82,287 & 584 & $312,082.50$ & 1.87 & $1.08(0.97-1.20)$ & \\
\hline 5 & 36,545 & 299 & $131,851.65$ & 2.27 & $1.31(1.15-1.50)$ & \\
\hline
\end{tabular}


Table 4. Cont.

\begin{tabular}{|c|c|c|c|c|c|c|}
\hline No. of MS Component & No. of Patients & Event & Duration & Rate & HR $(95 \%$ CI) & $p$-Value for Trend \\
\hline Prostate & & & & & & $<0.0001$ \\
\hline 0 & 112,376 & 456 & $594,275.88$ & 0.76732 & 1(Ref.) & \\
\hline 1 & 185,108 & 1127 & $975,527.38$ & 1.15527 & $1.50(1.35-1.68)$ & \\
\hline 2 & 198,662 & 1328 & $1,032,590.71$ & 1.28609 & $1.68(1.51-1.87)$ & \\
\hline 3 & 172,367 & 1270 & $882,278.37$ & 1.43945 & $1.90(1.70-2.11)$ & \\
\hline 4 & 125,359 & 1025 & $625,361.68$ & 1.63905 & $2.18(1.95-2.43)$ & \\
\hline 5 & 52,815 & 490 & $258,477.04$ & 1.89572 & $2.53(2.23-2.87)$ & \\
\hline Testicular & & & & & & 0.09 \\
\hline 0 & 112,376 & 10 & $595,563.22$ & 0.016791 & 1(Ref.) & \\
\hline 1 & 185,108 & 10 & $978,501.45$ & 0.01022 & $0.61(0.25-1.46)$ & \\
\hline 2 & 198,662 & 13 & $1,036,019.35$ & 0.012548 & $0.75(0.33-1.71)$ & \\
\hline 3 & 172,367 & 10 & $885,612.4$ & 0.011292 & $0.68(0.28-, 1.62)$ & \\
\hline 4 & 125,359 & 11 & $628,052.8$ & 0.017514 & $1.05(0.45-2.47)$ & \\
\hline 5 & 52,815 & 9 & $259,706.9$ & 0.034654 & $2.08(0.85-5.12)$ & \\
\hline
\end{tabular}

MS, Metabolic syndrome; HR, hazard ratio.

\section{Discussion}

In this study, we investigated the relationship between MS and malignancy, and determined the effect of individual MS components on malignancy in patients with HBV infection. We found that MS in HBV-infected individuals was associated with an increased risk of most malignancies, except for HCC. Additionally, most cancers showed an increase in prevalence as the number of MS components increased, however, this trend was reversed in HCC. Interestingly, high serum TG and low HDL-c levels were inversely associated with HCC risk.

HBV is a well-known cause of HCC. The integration of HBV DNA fragments into chromosomal DNA can result in the modulation of gene expression, especially of oncogenes and tumor suppressor genes in the liver. In recent studies, malignancies other than HCC have been shown to be associated with HBV infection $[9,10]$. In fact, HBV has been detected in several types of extrahepatic organ tissues, suggesting a potential role for HBV in the tumorigenesis of non-liver cancers [11]. Several studies have suggested that the $\mathrm{X}$ protein from HBV can bind and interfere with the components of DNA repair machinery and p53 in response to DNA damage, thereby increasing the risk of non-liver cancers [12,13].

It is generally accepted that metabolic disorders are closely associated with the occurrence and development of malignancies. However, there are insufficient data about the epidemiology of MS and the synergistic influence of MS on HCC and extrahepatic cancer development in patients with HBV. Some recent research has aimed to determine if the effect of individual components of MS were stronger than the overall effect of MS as a whole. For instance, in the case of endometrial cancer, obesity on its own carries a higher risk than MS as a whole [14]. As another example, while MS has been shown to be slightly associated with prostate cancer, a stronger association exists between prostate cancer and hypertension [15]. Therefore, assessment of individual MS components may help future therapies target the important factors of MS that contribute most to the development of malignancies. In the current study, MS tended to increase nearly all cancer risks in individuals with HBV infection, similar to the trend seen in the general population between MS and cancer more generally $[16,17]$. A higher number of MS components was associated with a significantly increased risk of all cancers types. However, our data show that HCC was inversely related to the presence of MS and the number of MS components. Interestingly, a low HDL-c and high level of TG were negatively correlated with the risk of HCC in the current study.

With regard to HCC, an association between MS and HCC has previously been demonstrated independent of the HBV state. The progression of hepatic fibrosis in relation to MS could result from the direct stimulation of liver stellate cells by hyperinsulinemia and 
hyperglycemia, resulting in an increased production of connective tissue growth factors and a subsequent accumulation of extracellular matrix. These processes may increase hepatic injury from other common factors, including HBV.

The role of lipid metabolism in cancer development has also not been fully explored to date. Although the exact mechanism on how HBV-related diseases are closely associated with lipid metabolisms has not been clarified until now, there are several other studies supporting our findings which show an inverse relationship between hypertriglyceridemia and the risk of HCC among those with HBV infections [18-20]. Lipid and lipoprotein metabolism are regulated by cytokines, and malignant hepatocyte cells are known to produce large amounts of pro-inflammatory cytokines. For instance, interleukin (IL)-6, tumor necrosis factor, and IL-1 may all inhibit TG synthesis [21]. The loss of the negative feedback mechanism for cholesterol regulation as well as an increase in cholesterol synthesis by undifferentiated HCC cells may be responsible for the hypercholesterolemia seen in cirrhotic patients with HCC [22].

In an experimental HBV X (HBx) transgenic mouse model, lipid metabolomics were analyzed during the progression of HBx transgenic HCC [23]. Serum TG was mildly increased during the early stage, but was decreased in mice with HCCs. The serum levels of cholesterol was mildly increased during the early stage and significantly increased during the HCC stage. The study by Frank et al. also identified that the dysregulation of lipid genes by HBV generates a series of molecular alterations which collectively activates oncogenes or inhibits tumor suppressors, thereby leading to tumorigenesis [23]. This study supports our data that the aberrant lipid metabolism with serum low TG and high cholesterol in individuals with HBV might be associated with HCC development.

Unfortunately, our study could not preclude the possibility that low TG was a consequence of the change in general nutritional status during disease progression and that high HDL-c was the result of statin use. Hence, a prospective study is needed to elucidate the causative and consequent effects of the abnormal lipid profile seen in HBV-related HCC.

Recently, a study on the association of metabolic risk factors with risks of cancer and all-cause mortality in patients with chronic hepatitis B was published using a similar but different dataset from the NHIS database. In the study by Lee et al. [17], the risk of developing HCC and non-HCC cancers both increased in patients with MS, which was different from the result of our study that showed negative association of HCC with the presence of MS. There could be several reasons for this discrepancy. First, while we analyzed the lipid profile after subdividing it into TG and HDL-c, Lee et al. used only total serum cholesterol level. Second, the waist circumference was substituted for BMI as one of the components of MS in their study, whereas we adopted the classic definition of MS. Third, the average age of the patients was younger (46 (37-54), median (IQR)) compared to our study population ( $53.5 \pm 9.77$, mean $\pm \mathrm{SD})$, which could have limited the assessment of cancer development due to a comparatively younger study population. More importantly, the number of patients lost to follow-up was over $50 \%$ in their study which could have acted as a shortcoming and rendered the data less representative of the population of interest.

Interestingly, the study by Chen et al. [24] demonstrated that the relationship between MS and the occurrence of HCC was negligible. This is based on the observation in the HBV endemic area that the link between MS and HCC via the development of NASH was trivial compared to the predominant influence of HBV infection per se on HCC development. However, the impact of MS on HCC was also insignificant in the population without HBV infection in their study, thus not being able to demonstrate the widely accepted MS-NASH-HCC sequence.

Differently from the study by Chen et al., the result of another study from Taiwan was quite similar to our study [18]. In this study, the authors demonstrated that DM was the most important risk factor for developing HCC in HBV carriers, and that central obesity was responsible for a $33 \%$ increase in the risk of HCC. It was also shown that 
hypertriglyceridemia reduced the risk of $\mathrm{HCC}$ by $32 \%$, which is in accordance with the finding of our study.

There are several limitations to our study. First, our study used retrospectively collected data from the NHIS database. Therefore, it is inevitable that there is missed information which may result in selection bias. However, this dataset comes from a nationwide population-based claims database with long-term follow-up of a large number of patients, thus reducing the potential for sampling bias. Second, since MS was determined by a single measurement at the time of the HCC screening program, changes in MS status over time were not available. Third, the status or activity of HBV infection and the usage of antiviral agents were not analyzed, which could have influenced the potential risk of HBV-related malignancy development. This is of particular importance in HCC as the associations between MS and liver disease can be influenced by the degree of underlying HBV-related liver pathology. In our dataset, there were no assessments of liver fibrosis either histologically, biochemically, or with transient elastography, which represents another significant limitation and provides a path for future study. Lastly, low TG and high HDL-c levels, which were associated with increased HCC risk, could have been attenuated by the presence of statin therapy. However, the Korean National Liver Cancer Surveillance Program database does not contain detailed information about self-administered medications.

In conclusion, the findings of this large population-based study indicate that MS in HBV carriers is associated with synergistic effect on the development of most malignancies, except for HCC. Moreover, the number of comorbid MS components was a risk factor for most cancers, but this trend was reversed in HCC. Finally, high serum TG and low HDL-c levels were inversely associated with HCC risk. This study could translate into the development of therapeutic strategies for MS by aiming to reduce cancer risk in patients with HBV infection. To date, the interactions between individual MS factors and HBV has yet to be fully elucidated. Pathophysiological studies are needed to better explore the possible biological mechanisms involved in the observed association between HBV infection and MS factors with regard to cancer development.

Supplementary Materials: The following are available online at https: / www.mdpi.com/article / 10.3390/jcm10112401/s1, Table S1: International Classification of Diseases, 10th revision (ICD-10) codes for non-HCC cancers

Author Contributions: Each author has been involved in and contributed to this manuscript. Conceptualization, J.W.C. and J.J.H.; Data curation, K.-D.H. and B.K.; Formal analysis, K.-D.H. and B.K.; Writing-original draft, J.W.C.; Writing-review \& editing, J.W.C. and J.J.H. All authors approved the final version of the manuscript to be published.

Funding: This research received no external funding.

Institutional Review Board Statement: The study was conducted according to the guidelines of the Declaration of Helsinki, and approved by the Institutional Review Board of Korea University Ansan Hospital (IRB number: AS0012, 21 January 2019).

Informed Consent Statement: Not applicable.

Data Availability Statement: Not applicable.

Acknowledgments: This study was supported by Korea University Ansan Hospital grant.

Conflicts of Interest: The authors declare no conflict of interest.

\section{References}

1. Hassan, M.M.; Hwang, L.Y.; Hatten, C.J.; Swaim, M.; Li, D.; Abbruzzese, J.L.; Beasley, P.; Patt, Y.Z. Risk factors for hepatocellular carcinoma: Synergism of alcohol with viral hepatitis and diabetes mellitus. Hepatology 2002, 36, 1206-1213. [CrossRef]

2. Welzel, T.M.; Graubard, B.I.; El-Serag, H.B.; Shaib, Y.H.; Hsing, A.W.; Davila, J.A.; McGlynn, K.A. Risk factors for intrahepatic and extrahepatic cholangiocarcinoma in the United States: A population-based case-control study. Clin. Gastroenterol. Hepatol. 2007, 5, 1221-1228. [CrossRef] [PubMed] 
3. Mason, A.; Wick, M.; White, H.; Perrillo, R.J.H. Hepatitis B virus replication in diverse cell types during chronic hepatitis B virus infection. Hepatology 1993, 18, 781-789. [CrossRef] [PubMed]

4. $\quad$ Lee, T.Y.; Lee, S.S.; Jung, S.W.; Jeon, S.H.; Yun, S.C.; Oh, H.C.; Kwon, S.; Lee, S.K.; Seo, D.W.; Kim, M.H.; et al. Hepatitis B virus infection and intrahepatic cholangiocarcinoma in Korea: A case-control study. Am. J. Gastroenterol. 2008, 103, 1716-1720. [CrossRef]

5. Inoue, M.; Kurahashi, N.; Iwasaki, M.; Tanaka, Y.; Mizokami, M.; Noda, M.; Tsugane, S.; Japan Public Health Center-based Prospective Study, G. Metabolic factors and subsequent risk of hepatocellular carcinoma by hepatitis virus infection status: A large-scale population-based cohort study of Japanese men and women (JPHC Study Cohort II). Cancer Causes Control 2009, 20, 741-750. [CrossRef]

6. Zhao, J.Y.; Zhao, Y.P.; Wang, H.; Gu, X.; Ji, J.; Gao, C.F. Association between metabolic abnormalities and HBV related hepatocelluar carcinoma in Chinese: A cross-sectional study. Nutr. J. 2011, 10. [CrossRef]

7. Wong, G.L.H.; Wong, V.W.S.; Choi, P.C.L.; Chan, A.W.H.; Chim, A.M.L.; Yiu, K.K.L.; Chan, H.Y.; Chan, F.K.L.; Sung, J.J.Y.; Chan, H.L.Y. Metabolic syndrome increases the risk of liver cirrhosis in chronic hepatitis B. Gut 2009, 58, 111-117. [CrossRef]

8. Lee, J.; Lee, J.S.; Park, S.H.; Shin, S.A.; Kim, K. Cohort Profile: The National Health Insurance Service-National Sample Cohort (NHIS-NSC), South Korea. Int. J. Epidemiol. 2017, 46, e15. [CrossRef] [PubMed]

9. Lok, A.S.F.; McMahon, B.J. Chronic hepatitis B. Hepatology 2007, 45, 507-539. [CrossRef]

10. Kamiza, A.B.; Su, F.H.; Wang, W.C.; Sung, F.C.; Chang, S.N.; Yeh, C.C. Chronic hepatitis infection is associated with extrahepatic cancer development: A nationwide population-based study in Taiwan. Bmc Cancer 2016, 16. [CrossRef]

11. Iloeje, U.H.; Yang, H.I.; Jen, C.L.; Su, J.; Wang, L.Y.; You, S.L.; Lu, S.N.; Chen, C.J. Risk of pancreatic cancer in chronic hepatitis B virus infection: Data from the REVEAL-HBV cohort study. Liver Int. 2010, 30, 423-429. [CrossRef] [PubMed]

12. Hsieh, A.; Kim, H.S.; Lim, S.O.; Yu, D.Y.; Jung, G. Hepatitis B viral X protein interacts with tumor suppressor adenomatous polyposis coil to activate Wnt/beta-catenin signaling. Cancer Lett. 2011, 300, 162-172. [CrossRef] [PubMed]

13. Xu, F.Q.; Zhu, X.M.; Han, T.; You, X.N.; Liu, F.B.; Ye, L.H.; Zhang, X.D.; Wang, X.H.; Yao, Y.Q. The oncoprotein hepatitis B $\mathrm{X}$-interacting protein promotes the migration of ovarian cancer cells through the upregulation of S-phase kinase-associated protein 2 by Sp1. Int. J. Oncol. 2014, 45, 255-263. [CrossRef]

14. Esposito, K.; Chiodini, P.; Capuano, A.; Bellastella, G.; Maiorino, M.I.; Giugliano, D. Metabolic syndrome and endometrial cancer: A meta-analysis. Endocrine 2014, 45, 28-36. [CrossRef] [PubMed]

15. Gacci, M.; Russo, G.I.; De Nunzio, C.; Sebastianelli, A.; Salvi, M.; Vignozzi, L.; Tubaro, A.; Morgia, G.; Serni, S. Meta-analysis of metabolic syndrome and prostate cancer. Prostate Cancer Prostatic Dis. 2017, 20, 146-155. [CrossRef]

16. Esposito, K.; Chiodini, P.; Colao, A.; Lenzi, A.; Giugliano, D. Metabolic syndrome and risk of cancer: A systematic review and meta-analysis. Diabetes Care 2012, 35, 2402-2411. [CrossRef]

17. Lee, Y.B.; Moon, H.; Lee, J.H.; Cho, E.J.; Yu, S.J.; Kim, Y.J.; Zoulim, F.; Lee, J.; Yoon, J.H. Association of Metabolic Risk Factors With Risks of Cancer and All-Cause Mortality in Patients With Chronic Hepatitis B. Hepatology 2020. [CrossRef]

18. Chen, C.L.; Yang, H.I.; Yang, W.S.; Liu, C.J.; Chen, P.J.; You, S.L.; Wang, L.Y.; Sun, C.A.; Lu, S.N.; Chen, D.S.; et al. Metabolic factors and risk of hepatocellular carcinoma by chronic hepatitis B/C infection: A follow-up study in Taiwan. Gastroenterology 2008, 135, 111-121. [CrossRef]

19. Motta, M.; Giugno, I.; Ruello, P.; Pistone, G.; Di Fazio, I.; Malaguarnera, M. Lipoprotein (a) behaviour in patients with hepatocellular carcinoma. Minerva Med. 2001, 92, 301-305.

20. Lagiou, P.; Kuper, H.; Stuver, S.O.; Tzonou, A.; Trichopoulos, D.; Adami, H.O. Role of diabetes mellitus in the etiology of hepatocellular carcinoma. J. Natl. Cancer Inst. 2000, 92, 1096-1099. [CrossRef]

21. Michiel, D.F.; Oppenheim, J.J. Cytokines as positive and negative regulators of tumor promotion and progression. Semin. Cancer Biol. 1992, 3, 3-15. [PubMed]

22. Ahaneku, J.E.; Taylor, G.O.; Olubuyide, I.O.; Agbedana, E.O. Abnormal lipid and lipoprotein patterns in liver cirrhosis with and without hepatocellular carcinoma. JPMA 1992, 42, 260-263.

23. Teng, C.F.; Hsieh, W.C.; Yang, C.W.; Su, H.M.; Tsai, T.F.; Sung, W.C.; Huang, W.; Su, I.J. A biphasic response pattern of lipid metabolomics in the stage progression of hepatitis B virus X tumorigenesis. Mol. Carcinog. 2016, 55, 105-114. [CrossRef] [PubMed]

24. Chen, C.T.; Chen, J.Y.; Wang, J.H.; Chang, K.C.; Tseng, P.L.; Kee, K.M.; Chen, P.F.; Tsai, L.S.; Chen, S.C.; Lin, S.C.; et al. Diabetes mellitus, metabolic syndrome and obesity are not significant risk factors for hepatocellular carcinoma in an HBV-and HCV-endemic area of Southern Taiwan. Kaohsiung J. Med. Sci. 2013, 29, 451-459. [CrossRef] [PubMed] 